TECHNICAL NOTE

\author{
A.C. Mamourian \\ K. Erkmen \\ D.J. Pluta
}

\section{Nonhelical Acquisition CT Angiogram after Aneurysmal Clipping: In Vitro Testing Shows Diminished Artifact}

\begin{abstract}
SUMMARY: Imaging of the cerebral vessels with use of CT angiogram (CTA) after placement of aneurysmal clips is often limited by clip artifacts. We used a phantom to demonstrate a visible reduction in metal artifact when using the axial technique, compared with the usual CTA helical acquisition. This approach may have some advantage when used for CTA with 64-section scanners in the specific circumstance of immediate postoperative imaging after placement of cerebral aneurysmal clips.
\end{abstract}

C T angiogram (CTA) is an attractive alternative for patients who require imaging of the cerebral vasculature after aneurysm clipping compared with conventional angiography. Although advances in CT technology and the use of titanium clips have improved the quality of CTA after aneurysmal clipping, artifacts arising from the clip limit the visualization of the aneurysmal neck and nearby vessels in some cases. ${ }^{1}$ CTA usually requires the use of helical image acquisition, to provide rapid imaging with sufficient coverage, but it is of interest to consider that CT imaging of the brain at most sites is done with the axial technique because, in principle, it has fewer artifacts. ${ }^{2}$ We wondered if axial imaging might improve the quality of CTA after placement of aneurysmal clips. This report describes the results of our study with use of a clipped aneurysmal phantom in which we compared the artifacts using the helical and axial techniques.

\section{Case Report/Technique}

We studied silicone models of aneurysms in both a simple water bath and inside a skull to simulate the beam hardening that occurs in vivo. We immersed the skull in a bucket of tap water for imaging. These silicon phantoms were filled with dilute contrast ( $1 \mathrm{~mL}$ of Omnipaque 350 [GE Healthcare, Piscataway, NJ] mixed in $9 \mathrm{~mL}$ of saline). We studied all of the aneurysmal phantoms with a 64-section scanner (GE Healthcare, Milwaukee, Wis) using our postclipping CTA technique (ie, $\mathrm{kV}, 140 ; \mathrm{mA}, 380$; section collimation, $0.625 \mathrm{~mm}$; pitch, 0.96; rotation speed, 0.4 seconds). These were compared with scans with use of the axial technique with a single rotation with 32 detectors collimated to $0.625 \mathrm{~mm}$ with a total coverage of $2 \mathrm{~cm}$. Images were reconstructed for both techniques at a $0.625-\mathrm{mm}$ interval (no overlap).

We used commercially available titanium and cobalt alloy clips. Titanium is known to have fewer artifacts on CT than cobalt alloy clips. $^{3}$ A Yasargil (Aesculap, Center Valley, Pa) 7-mm curved blade titanium clip and a 6-mm curved Sugita (Mizuho America, Beverly, Mass) cobalt alloy clip were positioned on the neck of the phantom aneurysm for the testing in the skull, and a titanium Sugita 7-mm blade straight clip was used for the experiments in the water bath.

Received May 29, 2007; accepted after revision August 14

From the Departments of Radiology (A.C.M., D.J.P.) and Neurosurgery (K.E.), DartmouthHitchcock Medical Center, Lebanon, NH.

Please address correspondence to Alexander C. Mamourian, MD, Dartmouth-Hitchcock Medical Center, One Medical Center Drive, Lebanon, NH 03756; e-mail: alexander. mamourian@uphs.upenn.edu

DOI 10.3174/ajnr.A0893
Test 1. We compared the images of a phantom 10-mm aneurysm in a water bath that had been clipped with a single titanium Sugita clip using both helical and axial imaging techniques.

Test 2. The silicon aneurysm model in the skull was imaged with 1 Sugita cobalt alloy clip positioned on the neck of the aneurysm. We obtained axial and helical images without moving the phantom or clip.

Test 3. A Yasargil titanium clip was positioned across the neck of a silicon aneurysm in the skull. This was imaged with use of the helical CTA and axial techniques. For the axial images, the tube rotation was varied from $0.4,1.0$, and 2 seconds with all other parameters unchanged. The images were reviewed on a workstation that allowed $3 \mathrm{D}$ imaging. These were all thresholded to the same window and level for visual comparisons.

\section{Results}

Test 1. On the water bath study, there was an obvious difference in the appearance of the clip artifact with a more compact artifact apparent on the axial scan of this titanium clip. Both the axial and helical scans used a 0.4-second tube rotation.

Test 2. On the images of the cobalt alloy clip taken through a skull, there was, again, a clear difference in the appearance of the artifact arising from the clip between the axial and helical acquisitions. The helical examination had many more visible white streaks arising from the aneurysm clip compared with the equivalent axial scan.

Test 3. The images of the titanium clip, again, showed less artifact on the axial scan compared with the helical source images (Fig 1). The 3D models acquired from the data revealed much sharper margins of the clip with use of axial imaging than helical images for the source data (Fig 2). Differences in image quality related to the rotation speed of the tube in axial mode were not apparent on the 3D models.

On the basis of these tests, we used axial imaging for the follow-up CTA of a patient 1 day after successful clipping of an anterior communicating aneurysm using a titanium clip (Yasargil). We established the timing for acquisition of the CTA using a timing bolus of $15 \mathrm{~mL}$ of contrast injected while scanning a single level in the neck at the level of C4. A cursor was placed within the high cervical internal carotid artery, and the time to peak attenuation was displayed as a graph on the operator console. The time delay to acquire the scan from the start of the injection was calculated to be the time-to-peak attenuation of the bolus plus 1 second. The $2-\mathrm{cm}$ imaging slab (defined by the center as 32 detectors on the 64-section scan- 

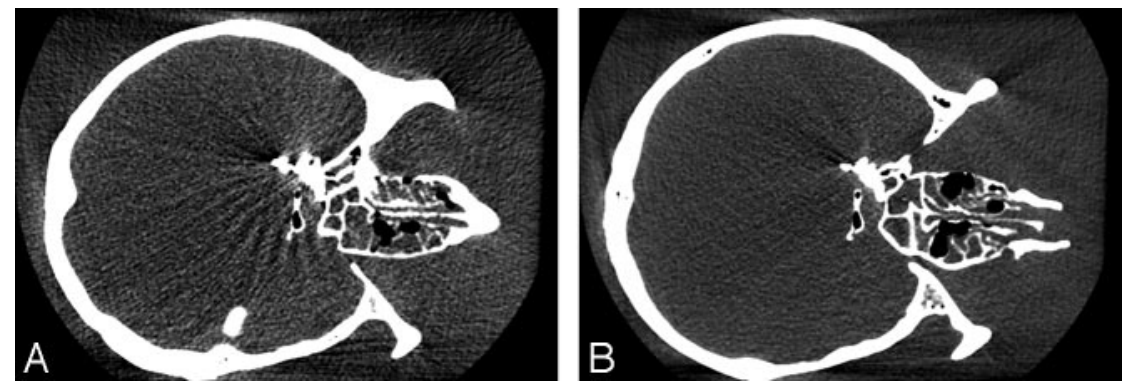

Fig 1. The helical acquisition of the titanium clip $(A)$ shows the lines of alternating white and dark arising from the clip. The axial scan done with an otherwise identical technique $(B)$ shows a diminished artifact in comparison to the helical acquisition.
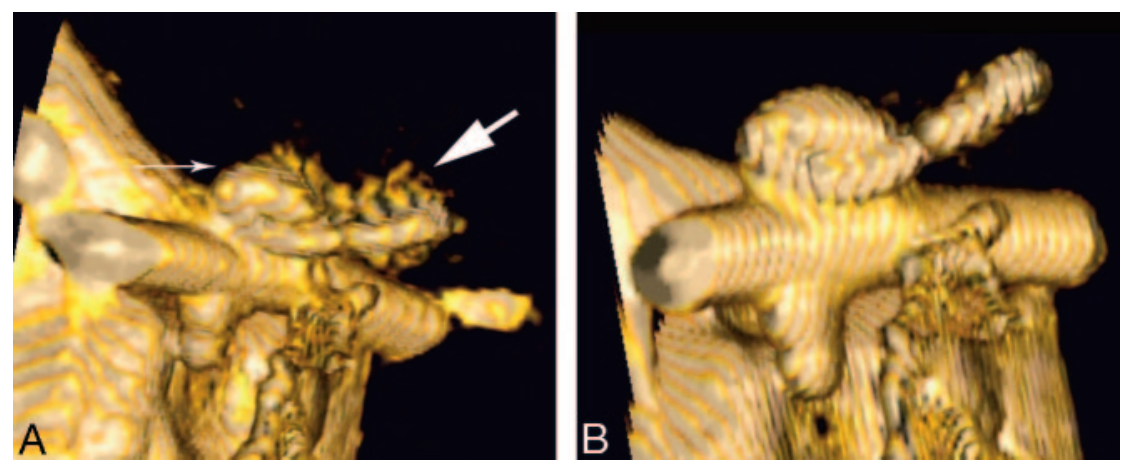

Fig 2. $A$, This $3 D$ reformatted image with use of a Vitrea workstation shows blurring of the margins of the aneurysmal clip on the side of the clip (large arrow). The aneurysma dome is visible (small arrow). The axial acquisition $(B)$ more clearly demonstrates the aneurysmal clip, with fewer artifacts arising from the margins of the clip, particularly at the level of the spring.
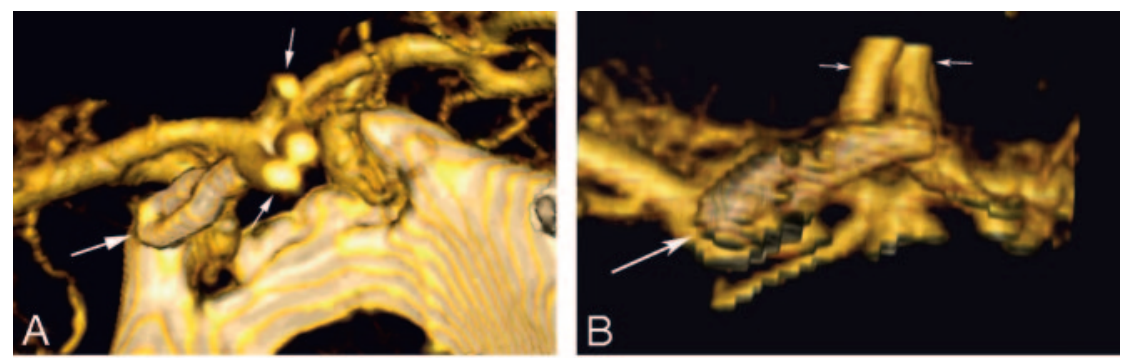

Fig 3. These $3 \mathrm{D}$ models were generated from the axial CTA acquisition. Images of the clip viewed from superior to inferior $(A)$ and inferior to superior $(B)$ show the relationship of the clip (large white arrow) to the A2 segments of the anterior cerebral arteries (small arrows). The maximum intensity projection image in the coronal plane $(C)$ again demonstrates the relationships of the clip (large arrow) to the $A 2$ segments (small arrows) and also highlights the narrow coverage of the axial acquisition while still including the pertinent anatomy around the clip and vessels distal to the clip.

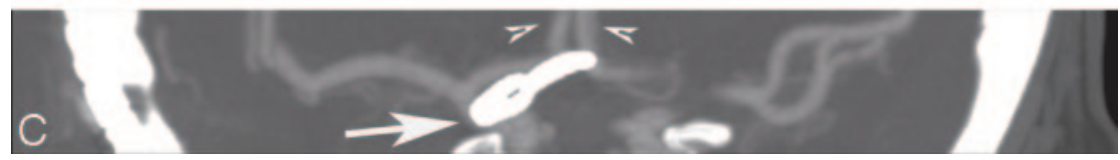

ner) was centered on the clip, which was easily identified on the scout. We used $100 \mathrm{cc}$ of contrast for this examination, though in principle it could be obtained with less.

Comparing the radiation dose and contrast requirements of this technique to conventional angiography is difficult because the radiation dose from conventional angiography is dependent on the hardware (eg, units with image intensifiers may deliver a larger dose than those by using digital flat plate) and the number of projections obtained. Some angiographers use rotational angiography to allow the generation of a $3 \mathrm{D}$ model as well as minimizing any need to acquire additional views. The contrast requirements for axial CTA and rotational angiography should prove to be comparable. Any comparison of these techniques must consider the relative procedure related risks as well as the speed of acquisition of the CTA.

We judged the image quality in our clinical case as excellent, with little visible artifact near the clip on source images and clear visualization of the aneurysm clip and adjacent vessels on the reformatted images and 3D reconstructions (Fig 3). The interpretation of an intraoperative conventional angiogram, which had been obtained at the time of clip placement, was in agreement (ie, there was no residual aneurysmal neck). Reviewing the dose reports calculated from the scanner for the preoperative CTA compared with the postoperative axial examination, while the CTDIvol was slightly higher for the axial scan (111 vs $76 \mathrm{mGy}$ ), we found that the dose length product was significantly less (223 mGy-cm vs $1264 \mathrm{mGy}-\mathrm{cm}$ ) because of the limited coverage needed for this postoperative study.

\section{Discussion}

Mutidetector scanners can be used to acquire images in either an axial or helical mode. One specific artifact associated with helical scanning has been compared with the blades of a windmill and is attributed to the difficulties of reconstructing a single section from data acquired from multiple detectors. ${ }^{2}$ The number of blades increases with pitch, and this might account for the decreased artifact noted by van der Schaaf et $\mathrm{al}^{4}$ when they used pitch of less than 1 in their phantom study of clip artifacts.

The goals of postoperative imaging the aneurysmal clip site immediately after placement of an aneurysmal clip is to establish the patency of the nearby vessels and the complete elimination of the aneurysm from the cerebral circulation. Intraoperative angiography is valuable because there is still the option of improving the clip position, but because of the limitations imposed by the operating room environment and lesser image 
quality compared with a dedicated angiography room, a second study in the immediate postoperative period is often warranted. ${ }^{5}$ This approach with axial CTA but with limited coverage would be well suited for immediate postoperative imaging and has the potential to eliminate the need for a postoperative conventional angiogram. Although this approach does require accurate determination of the scan coverage, this can be easily done with use of the clip itself based on the scout view and would not require physician interaction. The speed of the single section is sufficiently fast that several scans could be obtained at that same level to eliminate the possibility of missing the bolus though at the cost of additional radiation. The axial technique might be well suited for those cases with multiple clips in which conventional CTA is frequently limited by the composite artifacts. There may also be advantages to this approach in cases in which the clip is in close proximity to the skull, as is common with aneurysms of the anterior communicating artery and posterior inferior cerebellar artery. Follow-up angiography at 3 years or more should also allow detection of new aneurysms at remote sites, ${ }^{6}$ and, for this reason, helical CTA would be necessary.

The clip artifact would be minimized when the clip is oriented perpendicular to the section. This is more dependent on the orientation of the aneurysm and the approach used by the surgeon than it is on the actual aneurysm site, but it is more common to see transverse clip placement at all sites because of the lateral exposure commonly used.

Further evaluation of this approach is necessary because the factors that lead to metal artifacts are complex, and many are specific to scanners and reconstruction software. Currently, the use of titanium clips is implicit for any reasonable imaging of aneurysmal clips with CTA regardless of the scanner. This approach would not be feasible with an 8 -section scanner, however, because of limited coverage with thin sections. A scanner with more detectors than 64 should provide even more coverage with a single spin of the tube and may be the ideal for CTA for any indication. Clinical studies will be needed to determine whether this approach improves CTA sufficiently to justify the reduced coverage, but it would seem to be a promising approach based on this in vitro investigation.

\section{References}

1. Dehdashti AR, Binaghi S, Uske A, et al. Comparison of multislice computerized tomography angiography and digital subtraction angiography in the postoperative evaluation of patients with clipped aneurysms. J Neurosurg 2006;104:395-403

2. Barrett JF, Keat N. Artifacts in CT: recognition and avoidance. Radiographics 2004;24:1679-91

3. Lawton MT, Ho JC, Richard WC, et al. Titanium aneurysm clips: Part I-Mechanical, radiological, and biocompatibility testing. Neurosurgery 1996; 38:1158-63; discussion 1164

4. van der Schaaf I, van Leeuwen M, Vlassenbroek A, et al. Minimizing clip artifacts in multi CT angiography of clipped patients. AJNR Am J Neuroradiol 2006;27:60-66

5. Tang G, Cawley CM, Dion JE, et al. Intraoperative angiography during aneurysm surgery: a prospective evaluation of efficacy. J Neurosurg 2002;96:993-99

6. David CA, Vishteh AG, Spetzler RF, et al. Late angiographic follow-up review of surgically treated aneurysms. J Neurosurg 1999;91:396-401 